\title{
Highlight on Multidrug Resistance of Enterococcus faecalis Recovered from Diabetic Foot Patients
}

\author{
Ahmad ALbeloushi, ${ }^{1,2 *}$, Ayman Elbehiry ${ }^{3,4}$, Eman Marzouk ${ }^{5}$, Rasha Zahran ${ }^{3}$ and \\ Husam Edrees ${ }^{4}$
}

${ }^{1}$ Al Bukayriyah General Hospital, Qassim, Kingdom of Saudi Arabia. ${ }^{2}$ Department of Botany and Microbiology, College of Science, King Saud University, P.O. Box 2455, Riyadh 11451, Saudi Arabia. ${ }^{3}$ Department of Bacteriology, Mycology and Immunology, Faculty of Veterinary Medicine, University of Sadat City, Sadat, Egypt. ${ }^{4}$ Department of Public Health, College of Public Health and Health Informatics, Qassim University, Buraidah, Saudi Arabia. ${ }^{5}$ Department of Medical Laboratories, College of Applied Medical Sciences, Qassim University, Saudi Arabia.

\begin{abstract}
Diabetic foot infections (DFIs) are a progressively serious health problem worldwide. Enterococcus faecalis (E. faecalis) is one of the most frequent bacteria in DFIs. The antibiotic resistance patterns of this bacterium remain a significant tool for monitoring infection. Therefore, our study aimed to determine the susceptibility of $E$. faecalis recovered from the wounds of hospitalized diabetic foot patients to various antimicrobial drugs. Fifty-two $E$. faecalis strains were recovered from 630 diabetic foot patients. All isolates were identified biochemically by a Vitek ${ }^{\circledR} \mathbf{2}$ system and via a mass spectrometer (MALDI Biotyper). Antimicrobial sensitivity testing used Vitek 2 cards and Kirby-Bauer as the reference method. The findings indicated that the susceptibility of $E$. faecalis was $100 \%$ for ampicillin, ampicillin-sulbactam, benzylpenicillin, norfloxacin, and ofloxacin; $92 \%$ for nitrofurantoin, teicoplanin, and vancomycin; $\mathbf{8 7 \%}$ for imipenem; $\mathbf{8 1 \%}$ for kanamycin (high concentration) and tetracycline; $73 \%$ for levofloxacin; and $\mathbf{5 2 \%}$ for streptomycin (high concentrations). The resistance was $\mathbf{1 0 0 \%}$ for clindamycin and quinupristin-dalfopristin, $\mathbf{9 6 \%}$ for cefuroxime, $\mathbf{9 0 \%}$ for ciprofloxacin and erythromycin, $\mathbf{8 6 \%}$ for trimethoprim-sulfamethoxazole, $\mathbf{5 4 \%}$ for gentamicin (high concentration), and $\mathbf{4 8 \%}$ for streptomycin (high concentration). All E. faecalis strains were resistant against numerous antibiotics with a multiple antibiotic resistance (MAR) index of $0.20-0.60$. The mean value of MAR indices for all tested $E$. faecalis species was 0.373 . The high levels of antimicrobial resistance patterns to $E$. faecalis seen here are important because they restrict treatment possibilities and adversely affect the health of diabetic foot patients. Consequently, our findings should be carefully considered in public health and awareness programs.
\end{abstract}

Keywords: Diabetic foot infections; Enterococcus faecalis; Antibiotic resistance.

*Correspondence: aalbeloushi@yahoo.com; 00966503140996

(Received: 06 November 2018; accepted: 09 December 2018)

Citation: Ahmad ALbeloushi, Ayman Elbehiry, Eman Marzouk, Rasha Zahran and Husam Edrees, Highlight on Multidrug Resistance of Enterococcus faecalis Recovered from Diabetic Foot Patients, J Pure Appl Microbiol., 2019; 13(1):481-489 doi: 10.22207/ JPAM.13.1.54

C The Author(s) 2019. Open Access. This article is distributed under the terms of the Creative Commons Attribution 4.0 International License which permits unrestricted use, sharing, distribution, and reproduction in any medium, provided you give appropriate credit to the original author(s) and the source, provide a link to the Creative Commons license, and indicate if changes were made. 


\section{INTRODUCTION}

Diabetic foot (DF) is a chronic form of diabetes mellitus (DM) associated with high economic and social problems worldwide ${ }^{1,2}$. Approximately $15 \%$ of all diabetic patients eventually have a foot ulcer that is highly susceptible to bacterial infections ${ }^{3}$. Diabetic foot infections (DFIs) are particularly concerning due to the emergence of antibiotic-resistant bacteria ${ }^{4}$. There is currently a shortage of data on casualties of DF-particularly in the Middle East.

Saudi Arabia is a top ten Middle Eastern/ Arab countries in terms of diabetes prevalence in adults. This leads to high rates of foot ulcers along with increasing morbidity and costs ${ }^{5}$. Foot ulcers in diabetic patients are more susceptible to various microbial contaminations. These can spread rapidly and often lead to permanent tissue damage. Several bacteria can cause DFIs: Non-spore forming Gram-positive cocci (e.g., Enterococci) are the most common bacteria ${ }^{2,6}$.

Previous studies have shown that the Enterococcus genus is a main cause of the increase in the rate of morbidity and mortality in DFls ${ }^{7}$. This genus is composed of 38 species; Enterococcus faecalis (E. faecalis) is particularly common and often implicated in the transfer of antimicrobial resistance ${ }^{8,9}$. The clinical significance of $E$. faecalis is often associated with its antimicrobial resistancethis leads to problems with colonization and infection ${ }^{10}$.

One of the biggest problems facing diabetic foot patients is the isolation of a large number of microbes' that are resistant to various antibioticsespecially vancomycin-resistant Enterococci and methicillin-resistant Staphylococcus aureus ${ }^{2}$. The increased frequency of Enterococcus in DFIs is a main cause of hospitalization in Saudi hospitals perhaps because of increased antibiotic use. The presence of antibiotic-resistant bacteria highlights the importance of antimicrobial vulnerability testing for diabetic foot patients and the need to avoid excessive use of antimicrobials ${ }^{4,11}$.

Recently, VITEK 2 cards have been approved by the Food and Drug Administration (FDA) for antimicrobial susceptibility testing. This approach is fast, automatic, sensitive, and highly specific $^{12}$. Therefore, the suitable management of E. faecalis infections can lead to proper antibiotic choice based on susceptibility test reports ${ }^{11}$.
Primary management includes empirical antibiotic treatment based on local epidemiological data on antimicrobial susceptibility. Information on the microorganisms underlying the infections is critical to determining the appropriate antibiotic therapy ${ }^{3}$. Therefore, this study examined the antimicrobial susceptibility and resistance patterns of $E$. faecalis isolated from ulcers of diabetic foot patients in two hospitals in central Saudi Arabia.

\section{MATERIALS AND METHODS Bacterial strains}

We used 52 E. faecalis strains recovered from 630 samples collected from diabetic foot patients in the Bukyriah General Hospital (BGH), Al-Qassim region and King Saud Medical City Riyadh from June 2016 to January 2017.

\section{Identification of $E$. faecalis}

The Vitek 2 system (Biom'rieux, France) measured the biochemical profiles of $E$. faecalis isolates based on the manufacturer's instructions. American Type Culture Collection (ATCC) 19433 $E$. was used as the quality control. The Microflex LT (Bruker Daltonik, Bremen, Germany) was then applied for accurate identification of $E$. faecalis isolates. All procedures and data analysis were performed according to the recommendations provided by Bruker Daltonics Corporation. Escherichia coli was used as bacterial test standard (positive control). Genetic analysis detected the presence of $E$. faecalis virulence genes specific to $E$. faecalis. First, genomic DNA extraction was achieved by QuickGene-810 (Fujifilm, Tokyo, Japan). Second, six primer sets specific for $E$. faecalis including asa1, GeIE, cylA, esp, hy1, VanA and, Van $B$ were amplified using the SYBER Green RT-PCR (Applied Biosystems, USA).

Antibiotic susceptibility and MAR index of $E$. faecalis using Vitek 2 cards

We used the VITEK 2 AST-P516 cards (BioM rieux) to detect the susceptibility percentage of $E$. faecalis against various antimicrobial drugs. Each card consists of 64 holes containing 20 antibiotics at different concentrations (Table 1). In brief, 2-3 distinct colonies were suspended in sterilized physiological saline and thoroughly mixed. The McFarland turbidity was adjusted from 0.52 and 0.65 by DensiChekTM (BioMe2 rieux, France). Of this suspension, $5 \mathrm{ml}$ was loaded onto the AST-P516 cards. The filled cassette was placed in 
the device, and the results were interpreted by the AST-P516 database after an incubation period of $4 \mathrm{~h}$. Likewise, the multiple antibiotic resistance (MAR) index of each isolate was recorded through the calculation designated as follows:

MAR index $=\frac{\text { Number of antimicrobial agents to which the bacterium is resistant }}{\text { Total number of antimicrobial agents used in the study }}$

\section{Kirby-Bauer as a reference method}

The susceptibility of E. faecalis to various antibiotics was measured via the Kirby-Bauer method according to Clinical and Laboratory Standards Institute guidelines ${ }^{13}$. The results were sensitive, intermediate, or resistant according to the diameter of the inhibitory zones using CLSI breakpoints. The concentration ranges $(\mu \mathrm{g} / \mathrm{ml})$ of the antimicrobial agents and breakpoints used in antibiotic susceptibility test were demonstrated in Table 1. E. faecalis ATCC 29212 was used as a quality control bacterium for all tests ${ }^{14}$.

\section{RESULTS}

\section{Frequency and identification of $E$. faecalis}

The occurrence of E. faecalis was studied in 630 patients suffering from diabetic foot ulcers. Our findings revealed that 74 samples were positive to various types of bacteria including fifty twoE. faecalis, eight Acinetobacterbaumanni,

Table 1. Concentration range $(\mu \mathrm{g} / \mathrm{ml})$ of antimicrobial agents and breakpoints used in antibiotic susceptibility test

\begin{tabular}{|c|c|c|c|c|}
\hline \multirow[t]{2}{*}{ Antimicrobial agent } & \multicolumn{2}{|c|}{ MIC $(\mu \mathrm{g} / \mathrm{ml})$ range } & \multicolumn{2}{|c|}{ Breakpoint $^{\mathrm{a}}(\mu \mathrm{g} / \mathrm{ml})$} \\
\hline & Vitek 2 System & Kirby-Bauer & Susceptible & Resistant \\
\hline Ampicillin & $0.5-32$ & $0.015-32$ & $\leq 8$ & $\geq 16$ \\
\hline Ampicillin-sulbactam & $2-64$ & $0.015-32$ & $\leq 8$ & $\geq 16$ \\
\hline Benzylpenicillin & $0.125-64$ & $0.25-16$ & $\leq 8$ & $\geq 16$ \\
\hline Cefuroxime & 4-8 & $0.015-16$ & $\leq 4$ & $\geq 8$ \\
\hline Ciprofloxacin & $1-4$ & $0.12-4$ & $\leq 1$ & $\geq 4$ \\
\hline Clindamycin & $0.5-2$ & $0.015-32$ & $\leq 0.5$ & $\geq 4$ \\
\hline Erythromycin & $0.25-2$ & $0.015-32$ & $\leq 0.5$ & $\geq 8$ \\
\hline Gentamicin, high level & 150 & 500 & $\leq 500$ & $>500$ \\
\hline Kanamycin, high level & 200 & $128-1024$ & $\leq 512$ & $>1024$ \\
\hline Streptomycin, high level & 200 & 2000 & $\leq 1000$ & $\geq 1000$ \\
\hline Imipenem & $8-32$ & $0.015-16$ & $\leq 2$ & $\geq 8$ \\
\hline Levofloxacin & $0.25-8$ & $0.015-32$ & $\leq 2$ & $\geq 8$ \\
\hline Nitrofurantoin & $16-64$ & $2-64$ & $\leq 32$ & $\geq 128$ \\
\hline Norfloxacin & $0.5-4$ & $0.03-16$ & $\leq 2$ & $\geq 16$ \\
\hline Ofloxacin & $0.5-4$ & $0.03-64$ & $\leq 2$ & $\geq 4$ \\
\hline $\begin{array}{l}\text { Quinupristin- } \\
\text { Dalfopristin }\end{array}$ & $0.25-2$ & $0.5-32$ & $\leq 1$ & $\geq 4$ \\
\hline Teicoplanin & $1-16$ & $0.015-16$ & $\leq 8$ & $\geq 32$ \\
\hline Tetracycline & $0.5-2$ & $0.03-16$ & $\leq 4$ & $\geq 16$ \\
\hline $\begin{array}{l}\text { Trimethoprim- } \\
\text { sulfamethoxazole }\end{array}$ & $160-640$ & $20-40$ & $\leq 2 / 38$ & $\geq 4 / 76$ \\
\hline Vancomycin & $2-6$ & $0.015-16$ & $\leq 4$ & $>32$ \\
\hline
\end{tabular}

a means breakpoints of various antibiotics tested by Kirby-Bauer as described by Clinical \& Laboratory Standards Institute (CLSI)

four Staphylococcus aureus and two isolates for each Citrobacterfreundii, Klebsiella pneumoniae, Staphylococcus epidermidis, Enterobacter aerogenes and Escherichia coli. Through these results, it is clear that the E. faecalis is the most common recovered bacteria in DFIs. After biochemical identification, 49isolates of E. faecalis were recognized biochemically by Vitek ${ }^{\mathrm{TM}} 2$ compact system. Microflex LT results showed that a total of $52(100 \%)$ E. faecalis isolates were correctly identified at the species level as $44.23 \%(23 / 52)$ strains were appropriately recognized with a log of $2.3-3.0$, while $51.92 \%$ (27/52) were accurately well-known with a log 2.0-2.29. In contrast, only 
two isolates (3.84\%) were recognized at the genus level with a log score extending from 1.7 to 1.99 . Six virulence genes (asa1, GelE, cylA, esp, hy1, VanA, and $\operatorname{VanB}$ ) were detected in all E. faecalis isolates.

\section{Antibiotic Susceptibility and MAR of $E$. faecalis}

The minimum inhibitory concentrations (MICs) of 20 antibacterial drugs were detected for 52 E. faecalis isolates using Vitek 2 system cards via the Kirby-Bauer method. Table 2 and Fig. 1 show that the susceptibility of $E$. faecalis was $100 \%$ for ampicillin, ampicillinsulbactam, benzylpenicillin, norfloxacin, and ofloxacin; $92 \%$ for nitrofurantoin, teicoplanin, and vancomycin; $87 \%$ for imipenem; $81 \%$ for kanamycin (high concentrations) and tetracycline; 73\% for levofloxacin; and 52\% for streptomycin (high concentrations). The resistance was $100 \%$ for clindamycin and quinupristin-dalfopristin, $96 \%$ for cefuroxime, $90 \%$ for ciprofloxacin and erythromycin, $86 \%$ for trimethoprimsulfamethoxazole, $54 \%$ for gentamicin (high concentration), $48 \%$ for streptomycin (high concentration), $27 \%$ for levofloxacin, and $19 \%$ for kanamycin (high concentration).

Table 3 illustrates the MAR index of 52 E. faecalis strains in diabetic foot infections. The mean value of MAR index of all E. faecalis

Table 2. Susceptibility of $E$. faecalis against antimicrobial agents using Vitek 2 cards with a reference of Kirby-Bauer method

\begin{tabular}{lcccccccc}
\hline \multirow{2}{*}{ Antimicrobial agent } & \multicolumn{3}{c}{ VITEK 2 System } & \multicolumn{4}{c}{ Kirby-Bauer method } \\
& Susceptibility & \multicolumn{3}{c}{ Resistant } & Susceptibility & Resistant \\
& No. & $\%$ & No. & $\%$ & No. & $\%$ & No. & $\%$ \\
\hline Ampicillin & 52 & 100 & 0 & 0 & 52 & 100 & 0 & 0 \\
Ampicillin-sulbactam & 52 & 100 & 0 & 0 & 52 & 100 & 0 & 0 \\
Benzylpenicillin & 52 & 100 & 0 & 0 & 52 & 100 & 0 & 0 \\
Cefuroxime & 0 & 0 & 50 & 96 & 0 & 0 & 52 & 100 \\
Ciprofloxacin & 5 & 10 & 47 & 90 & 5 & 10 & 47 & 90 \\
Clindamycin & 0 & 0 & 52 & 100 & 0 & 0 & 52 & 100 \\
Erythromycin & 5 & 10 & 47 & 90 & 4 & 8 & 48 & 92 \\
Gentamicin, high level & 24 & 46 & 28 & 54 & 24 & 46 & 28 & 54 \\
Kanamycin, high level & 42 & 81 & 10 & 19 & 40 & 77 & 12 & 23 \\
Streptomycin, high level & 27 & 52 & 26 & 48 & 27 & 52 & 26 & 48 \\
Imipenem & 45 & 87 & 7 & 13 & 46 & 88 & 6 & 12 \\
Levofloxacin & 38 & 73 & 14 & 27 & 38 & 73 & 14 & 27 \\
Nitrofurantoin & 50 & 96 & 2 & 4 & 52 & 100 & 0 & 0 \\
Norfloxacin & 52 & 100 & 0 & 0 & 52 & 100 & 0 & 0 \\
Ofloxacin & 52 & 100 & 0 & 0 & 52 & 100 & 0 & 0 \\
Quinupristin-Dalfopristin & 0 & 0 & 52 & 100 & 0 & 0 & 52 & 100 \\
Teicoplanin & 48 & 92 & 4 & 8 & 52 & 100 & 0 & 0 \\
Tetracycline & 42 & 81 & 10 & 19 & 40 & 77 & 12 & 23 \\
Trimethoprim- & 7 & 14 & 45 & 86 & 6 & 11 & 46 & 89 \\
sulfamethoxazole & & & & & & & \\
Vancomycin & 48 & 92 & 4 & 8 & 48 & 92 & 4 & 8 \\
\hline & & & & & & & \\
\hline
\end{tabular}

isolates was 0.373. All E. faecalis strains are resistant against numerous antibiotics (Fig. 2); the MAR index ranges from $0.20-0.60$. Strain No.13 exhibited a high degree of resistance against 12 out of 20 antimicrobial agents (MAR index of 0.60 ) followed by strain Nos. 8 \& 17, which were resistant to 11 of the 20 antibiotics (MAR index of 0.55$)$. Strain Nos. 1, 2, 11, 14, 21, 40, \& 41 had a MAR index of 0.50 . Strain Nos. $33 \& 43$ had the lowest resistant among all tested E. faecalis strains with a MAR index of 0.20 . The percentage of $E$. faecalis strains with a MAR index $>0.2$ was $50 / 52(96.16 \%)$; the percentage of $E$. faecalis strains with a MAR index d" 0.2 was 2/52 (3.84\%). Consequently, E. faecalis is found to be extremely resistant to numerous antibiotics with high MAR indices. 
Table 3. Multi antibiotic resistance (MAR) patterns distribution among 52 E. faecalis isolates

\begin{tabular}{|c|c|c|}
\hline $\begin{array}{l}\text { No. of } \\
\text { strain }\end{array}$ & Antimicrobial resistance profile & MAR index \\
\hline 1 & CEF, CIP, CLI, ERY, GEN/HL, IMI, LEV,QUI/D, TEI, TRI/S & 0.50 \\
\hline 2 & CEF, CIP, CLI, ERY, GEN, IMI, LEV, QUI/D, TRI, VAN & 0.50 \\
\hline 3 & CEF, CIP, CLI, ERY, GEN, QUI/D, TET, TRI, VAN & 0.45 \\
\hline 4 & CEF, CIP, CLI, ERY, QUI/D, TET, TRI/S & 0.35 \\
\hline 5 & CEF, CIP, CLI, ERY, GEN/HL, QUI/D, TRI/S & 0.35 \\
\hline 6 & CEF, CIP, CLI, ERY, GEN/HL, LEV, QUI/D, TRI/S & 0.40 \\
\hline 7 & CEF, CIP, CLI, ERY, GEN/HL, IMI, LEV, NIT, QUI/D, TRI/S & 0.50 \\
\hline 8 & CEF, CIP, CLI, ERY, GEN/HL, IMI, LEV, NIT, QUI/D, TEI, TRI/S & 0.55 \\
\hline 9 & CEF, CIP, CLI, ERY, QUI/D, TRI/S & 0.30 \\
\hline 10 & CEF, CIP, CLI, ERY, GEN/HL, QUI/D, TRI/S & 0.30 \\
\hline 11 & CEF, CIP, CLI, ERY, GEN/HL, KAN/HL, STR/HL, QUI/D, TRI/S & 0.45 \\
\hline 12 & CEF, CIP, CLI, ERY, GEN/HL, KAN/HL, STR/HL, QUI/D, TET, TRI/S & 0.50 \\
\hline 13 & CEF, CIP, CLI, ERY, GEN/HL, KAN/HL, STR/HL, IMI, LEV, QUI/D, TEI, TRI/S & 0.60 \\
\hline 14 & CEF, CIP, CLI, ERY, GEN/HL, KAN/HL, STR/HL, LEV, QUI/D, TRI/S & 0.50 \\
\hline 15 & CEF, CIP, CLI, ERY, GEN/HL, KAN/HL, STR/HL, QUI/D, TRI/S & 0.45 \\
\hline 16 & CEF, CIP, CLI, ERY, GEN/HL, KAN/HL, STR/HL, QUI/D, TRI/S & 0.45 \\
\hline 17 & CEF, CIP, CLI, KAN/HL, STR/HL, IMI, LEV, QUI/D, TEI, TET, TRI/S & 0.55 \\
\hline 18 & CEF, CIP, CLI, ERY, STR/HL, QUI/D, VAN & 0.35 \\
\hline 19 & CEF, CIP, CLI, ERY, STR/HL, QUI/D, VAN & 0.35 \\
\hline 20 & CEF, CIP, CLI, ERY, KAN/HL, STR/HL, LEV, QUI/D, TRI/S & 0.45 \\
\hline 21 & CEF, CIP, CLI, ERY, GEN/HL, KAN/HL, STR/HL, IMI, LEV, QUI/D & 0.50 \\
\hline 22 & CEF, CIP, CLI, ERY, GEN/HL, KAN/HL, STR/HL, QUI/D, TRI/S & 0.45 \\
\hline 23 & CEF, CIP, CLI, QUI/D, TRI/S & 0.25 \\
\hline 24 & CEF, CIP, CLI, QUI/D, TRI/S & 0.25 \\
\hline 25 & CEF, CIP, CLI, ERY, QUI/D, TET, TRI/S & 0.35 \\
\hline 26 & CEF, CIP, CLI, ERY, QUI/D, TRI/S & 0.30 \\
\hline 27 & CEF, CIP, CLI, ERY, STR/HL, QUI/D, TEI, TET & 0.40 \\
\hline 28 & CEF, CIP, CLI, ERY, GEN/HL, STR/HL, QUI/D, TRI/S & 0.40 \\
\hline 29 & CEF, CIP, CLI, ERY, GEN/HL, STR, QUI/D, TRI/S & 0.40 \\
\hline 30 & CEF, CIP, CLI, ERY, GEN/HL, STR/HL, QUI/D, TRI/S & 0.40 \\
\hline 31 & CEF, CIP, CLI, ERY, GEN/HL, STR/HL, QUI/D, TRI/S & 0.40 \\
\hline 32 & CEF, CIP, CLI, ERY, GEN/HL, STR/HL, QUI/D, TRI/S & 0.40 \\
\hline 33 & CEF, CLI, STR/HL, QUI/D & 0.20 \\
\hline 34 & CEF, CIP, CLI, STR/HL, QUI/D & 0.25 \\
\hline 35 & CEF, CIP, CLI, ERY, STR/HL, QUI/D, TET, TRI/S & 0.25 \\
\hline 36 & CEF, CIP, CLI, ERY, STR/HL, TET, TRI/S & 0.35 \\
\hline 37 & CEF, CLI, ERY, GEN/HL, STR/HL, TRI/S & 0.30 \\
\hline 38 & CEF, CIP, CLI, ERY, GEN/HL, QUI/D, TRI/S & 0.35 \\
\hline 39 & CEF, CIP, CLI, ERY, GEN/HL, QUI/D, TRI/S & 0.35 \\
\hline 40 & CEF, CIP, CLI, ERY, GEN/HL, LEV, STR/HL, QUI/D, TET, TRI/S & 0.50 \\
\hline 41 & CEF, CIP, CLI, ERY, GEN/HL, LEV, STR/HL, QUI/D, TET, TRI/S & 0.50 \\
\hline 42 & CEF, CLI, ERY, QUI/D, TRI & 0.25 \\
\hline 43 & CLI, ERY, QUI/D, TRI & 0.20 \\
\hline 44 & CEF, CIP, CLI, ERY, QUI/D, TRI/S & 0.30 \\
\hline 45 & CEF, CIP, CLI, ERY, QUI/D, TRI/S & 0.30 \\
\hline 46 & CEF, CIP, CLI, ERY, QUI/D, TRI/S & 0.30 \\
\hline 47 & CEF, CIP, CLI, ERY, QUI/D, TRI/S & 0.30 \\
\hline 48 & CEF, CIP, CLI, ERY, QUI/D & 0.25 \\
\hline 49 & CIP, CLI, ERY, GEN/HL, LEV, QUI/D & 0.25 \\
\hline 50 & CEF, CLI, ERY, LEV, QUI/D, TRI/S & 0.30 \\
\hline 51 & CEF, CIP, CLI, ERY, QUI/D & 0.25 \\
\hline 52 & CEF, CIP, CLI, ERY, QUI/D & 0.25 \\
\hline
\end{tabular}

Ampicillin = AM; Ampicillin-sulbactam = AM/S; Benzylpenicillin = BP; Cefuroxime = CEF; Ciprofloxacin = CIP; Clindamycin = CLI; Erythromycin = ERY; Gentamicin high level = GEN/HL; Kanamycin, high level = KAN/HL; Streptomycin high level = STR/HL; Imipenem = IMI; Levofloxacin = LEV; Nitrofurantoin = NIT; Norfloxacin = NOR; Ofloxacin = OFL; Quinupristin-Dalfopristin = QUI/D; Teicoplanin = TEI; Tetracycline = TET; Trimethoprimsulfamethoxazole $=$ TRI/S; Vancomycin $=$ VAN 


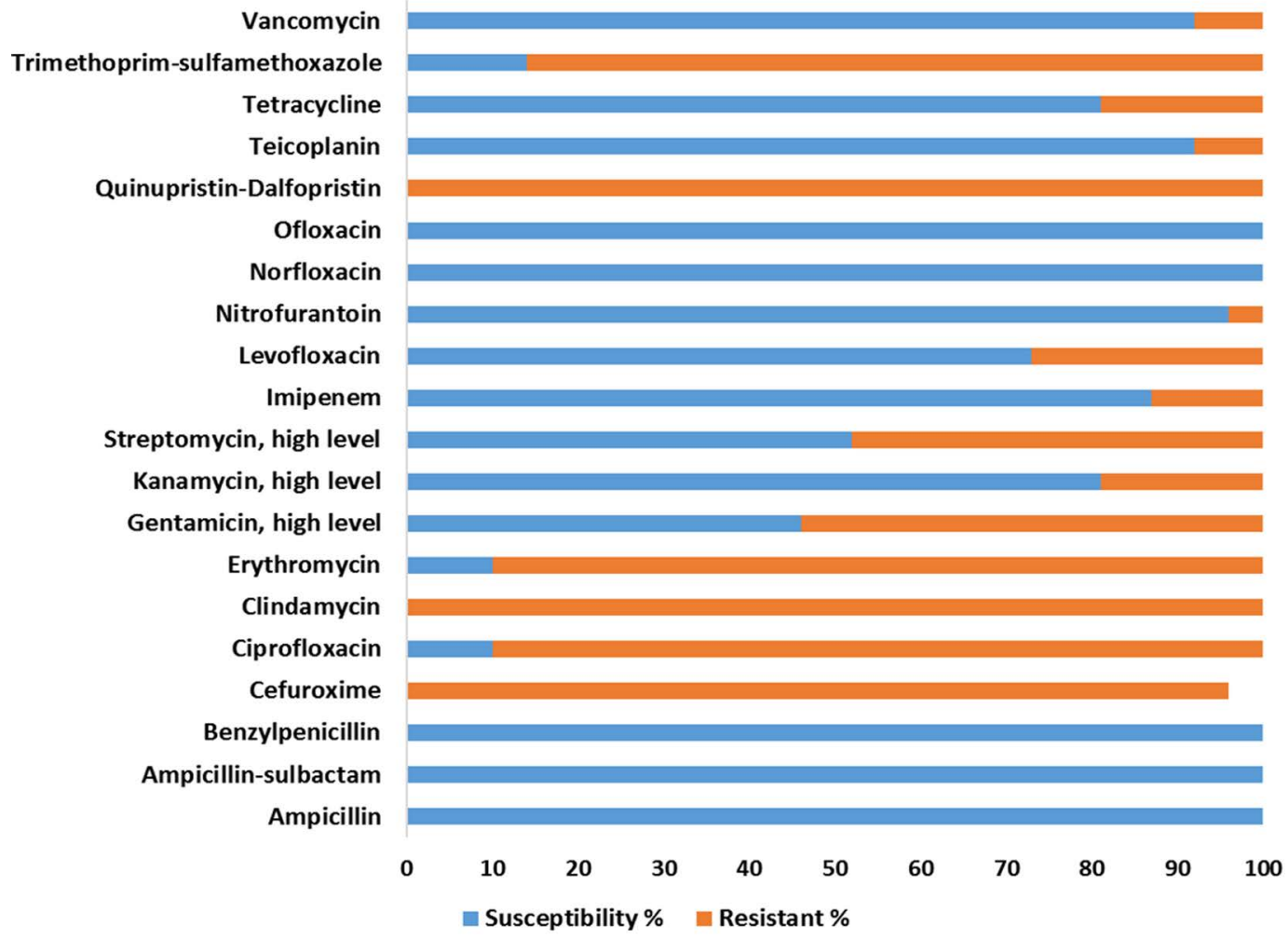

Fig. 1. Susceptibility and resistance percentage (\%) of E. faecalis against antimicrobial agents using Vitek 2 cards.

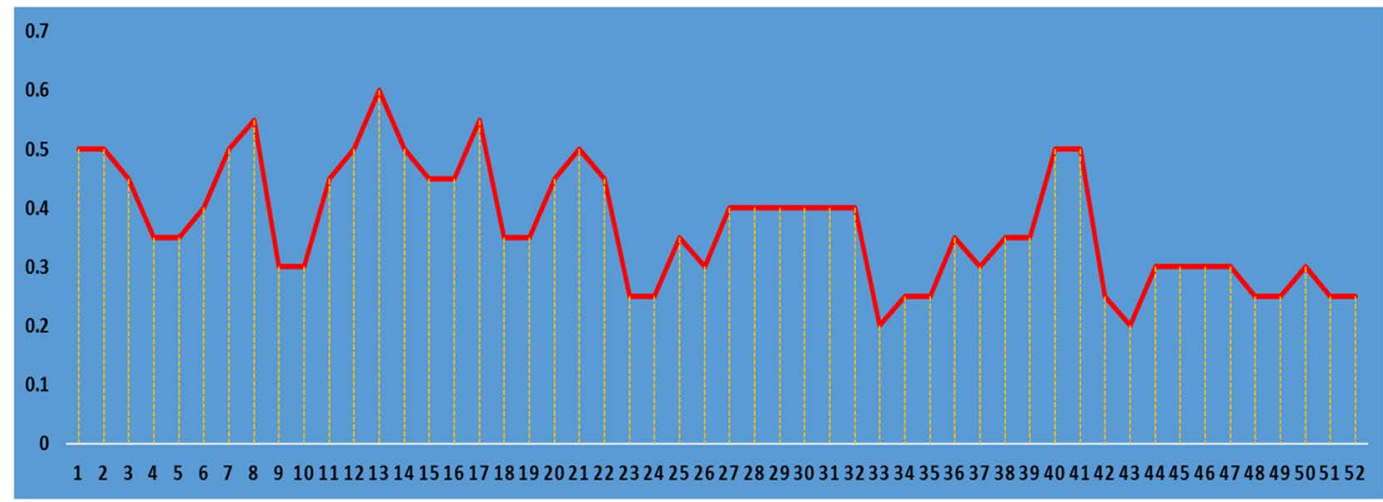

Fig. 2. Multi antibiotic resistance index (MAR index) patterns distribution among 52 E. faecalis isolates.

\section{DISCUSSION}

Most DFIs are polymicrobial and multidrug-resistant (MDR); the Enterococcus genus is an essential part of dangerous microbial milieu found in diabetic foot ulcers ${ }^{15,16}$. Previous work showed that the Enterococcus genus is one of the most important positive microorganisms isolated from diabetic foot patients. It significantly contributes to the increasing rates of morbidity and mortality from this disease ${ }^{7}$. Recently, Enterococcus species have been shown to be significant nosocomial pathogens, and E. faecalis and $E$. faecium isolates are the most common and virulent nosocomial microorganisms in many parts of the world ${ }^{17}$. 
The consequences of diabetic foot ulcers are very complex because the infection frequently becomes chronic and ultimately leads to increasing mortality rates. The misuse of antibiotics used in the treatment of DFls can increase the ability of bacteria to establish strong resistance against various antibiotics; thus, they adversely affect health due to treatment failure ${ }^{7}$. This study screened 630 samples from DFls for the presence $E$. faecalis, and 74 samples were positive: 52 E. faecalis, 8 Acinetobacter baumanni, 4 Staphylococcus aureus, 2 Citrobacter freundii, 2 Klebsiella pneumoniae, 2 Staphylococcus epidermidis, 2 Enterobacter aerogenes, and 2 Escherichia coli. These data confirm that $E$. faecalis is a keystone species in diabetic foot patients ${ }^{18}$.

The susceptibility data indicated that $100 \%$ of $E$. faecalis strains were highly sensitive to beta lactams (ampicillin \& ampicillin-sulbactam), penicillin (benzylpenicillin), and fluoroquinolone (norfloxacin and ofloxacin) groups; 92\% were sensitive to nitrofuran (nitrofurantoin) and glycopeptide (teicoplanin and vancomycin) groups; $87 \%$ were sensitive to the beta lactam (imipenem) group, $81 \%$ were sensitive to aminoglycosides (kanamycin, high concentration) and tetracycline, $73 \%$ were sensitive to fluoroquinolones (levofloxacin); and 52\% were sensitive to aminoglycoside (high concentration streptomycin). This finding suggests that these antimicrobial agents can be used for empirical treatment of $E$. faecalis infections. Similar results were previously reported by Dupreet et al. ${ }^{19}$ and Gopinat and Prakash ${ }^{20}$ who stated that E. faecalis isolates were susceptible to ampicillin, tigecycline, and teicoplanin. In addition, Wu et al. ${ }^{21}$ found that $E$. faecalis was most susceptible to ampicillin (100\%) followed by vancomycin (96.6\%), penicillin G (96.6\%), and linezolid (86.2\%).

One curious finding was that $E$. faecalis had multidrug resistance ( $\left(e^{\prime \prime} 4\right.$ and $\left.d^{\prime \prime} 12\right)$. Based on our interpretations, the resistance rate of $E$. faecalis isolates against various antimicrobial drugs were $100 \%$ for clindamycin and quinupristin-dalfopristin, $96 \%$ for cefuroxime, 90\% for ciprofloxacin and erythromycin, $86 \%$ for trimethoprim-sulfamethoxazole, $54 \%$ for gentamicin high level, $48 \%$ for streptomycin, high level, $27 \%$ for levofloxacin, $19 \%$ for kanamycin high level, and $13 \%$ for imipenem.
Similar results were obtained by Rams et al. ${ }^{22}$ who tested the susceptibility of 47 subgingival $E$. faecalis clinical isolates against various antimicrobial drugs. They found that the isolates had in vitro resistance to clindamycin $(100 \%$ resistant to $2 \mu \mathrm{g} / \mathrm{ml})$, erythromycin $(80.8 \%)$, and tetracycline (53.2\%). Jia et al. ${ }^{23}$ studied the resistance of Enterococcus species from a university hospital in China. They reported a higher frequency of tolerance to quinupristin/ dalfopristin, minocycline, chloramphenicol, and tetracycline in E. faecalis. A Portuguese study by Semedo-Lemsaddeket et al. ${ }^{7}$ also reported multi-drug resistance against Enterococcal species isolated from DF patients. In Brazil, Komiyama et al. ${ }^{24}$ found that an important proportion of the E. faecalis isolates recovered from oral biofilms were resistant to numerous antimicrobial drugsespecially to tetracycline, chloramphenicol, and erythromycin. Anvarinejad et al. ${ }^{25}$ isolated 34 Enterococcus species from 86 diabetic patients and found that $E$. faecalis was the most commonly isolated Enterococcus species (50\%). They also found that ciprofloxacin was the most resistant drug followed by gentamycin, imipenem, and vancomycin $(20.6 \%)$ against isolates.

Our findings for carbapenem (imipenem) resistance among $E$. faecalis (13\%) is incompatible with prior reports ${ }^{26}$, which showed that resistance rate of $E$. faecalis against other carbapenems (ertapenem) might be as high as $90 \%$. Despite the alarming resistance to vancomycin reported E. faecalis isolates, we found low resistance to vancomycin $(8 \% ; 4 / 52)$. Although the CLSI stated that Enterococcus species may be sensitive in vitro to various antimicrobial drugs such as cephalosporins, aminoglycosides, clindamycin, and trimethoprim-sulfamethoxazole, these antibiotics are not active clinically and would not be described as vulnerable ${ }^{27}$.

Data on the causative microbes (bacteria) in diabetic foot patients and their sensitivity to antibiotics is critical for the proper treatment and monitoring of infection ${ }^{28}$. The MAR status seen in the majority of the Enterococcus genus remains extremely significant-particularly in chronic and severe Enterococcal infections in DFIs-because antibiotic resistance frequently leads to treatment failure. The existence of MAR in diabetic foot ulcer Enterococci is an urgent matter because it can likely 
transfer that tolerance to other types of bacteria ${ }^{7}$. We found differences in the antimicrobial susceptibility and development of multi-drug resistance against $E$. faecalis as a function of location. This might be due to differences in drug prescription practices. Our results should be confirmed in a larger cohort because this is the first study to evaluate antimicrobial resistance profile against $E$. faecalis recovered from diabetic foot patients in Saudi Arabia.

\section{CONCLUSION}

Antimicrobial susceptibility testing of $E$. faecalis can help optimize the use of antimicrobials. The high levels of antimicrobial resistance patterns seen here in $E$. faecalis are of serious alarm because it limits treatment possibilities and adversely affects the health of affected diabetic foot patients. Consequently, our findings should be considered in public health strategies and awareness programs.

\section{ACKNOWLEDGEMENTS}

The authors gratefully acknowledge the King Abdulaziz City for Science and Technology, Saudi Arabia for funding this research (Project number 1-17-01-001-0054).

\section{CONFLICTS OF INTEREST}

The author declares that there are no conflict of interest.

\section{REFERENCES}

1. Doria M, Rosado V, Pacheco LR, Hernבndez M, Betriu A, Valls J, Franch-Nadal J, Fernבndez, Mauricio D. Prevalence of Diabetic Foot Disease in Patients with Diabetes Mellitus under Renal Replacement Therapy in Lleida, Spain. Biomed Res Int., 2016; 8:1-8.

2. Anvarinejad M, Pouladfar G, Japoni A, Bolandparvaz S, Satiary Z, Mardaneh J. Diabetic Foot Infections Antibiotic Susceptibility Patterns and Determination of Antibiotic Cross-Resistance inClinical Isolates of Enterococcus Species During 2012 - 2014 in Shiraz, Iran. Arch Pediatr Infect Dis., 2017;5:e37680.

3. Gadepalli R, Dhawan B, Sreenivas V, Kapil A, Ammini AC, Chaudhry R. A clinico microbiological study of diabetic foot ulcers in an Indian tertiary care hospital. Diabetes Care, 2006; 29:1727 1732.

4. Khoharo HK, Ansari S, Qureshi F. Diabetic foot ulcers: Common isolated pathogens and in vitro antimicrobial activity. Prof Med. J., 2009; 16:53 60.

5. Al Dawish MA, Robert AA, Braham R, Al Hayek AA, Al Saeed A, Ahmed RA, Al Sabaan FS. Diabetes Mellitus in Saudi Arabia: A Review of the Recent Literature. Curr
Diabetes Rev., 2016; 12:359-368.

6. Lipsky BA. Empirical therapy for diabetic foot infections: are there clinical clues to guide antibiotic selection? Clin Microbiol Infect, 2007;13:351-353.

7. Semedo-Lemsaddek T, Mottola C, Alves-Barroco C, Cavaco-Silva P, Tavares L, Oliveira M. Characterization of multidrug-resistant diabetic foot ulcer enterococci. Enferm Infecc Microbiol Clin., 2016; 34:114-116.

8. Fisher K, Phillips C. The ecology, epidemiology and virulence of Enterococcus. Microbiology, 2009; 155:1749-1757.

9. John VuCa, rvalho J. Enterococcus: review of its physiology, pathogenesis, diseases and the challenges it poses for clinical microbiology. J Front Biol., 2001; 6:357.

10. Furlaneto-maia L, Rocha K, Siqueira V, Furlaneto M. Comparison between automated system and pcrbased method for identification and antimicrobial susceptibility profile of clinical Enterococcus spp. Rev Inst Med Trop Sao Paulo., 2014; 56:97-103.

11. Mansilha A, BrandTo D. Guidelines for treatment of patients with diabetes and infected ulcers. J. Cardiovasc Surg (Torino), 2013; 54:193 200.

12. Cilloniz C, Martin-Loeches I, Garcia-Vidal C, San Jose A, Torres A. Microbial Etiology of Pneumonia: Epidemiology, Diagnosis and Resistance Patterns. Int. J. Mol Sci., 2016; 17:2120.

13. CLSI, Clinical and Laboratory Standards Insttute. Performance Standards for Antmicrobial Disk and Diluton Susceptbility Tests for Bacteria Iso- lated from Animals", Approved Standards - (4th ed.). CLSI document VET01-A4, Wayne, PA, USA 2016.

14. Khalili H, Soltani R, Negahban S, Abdollahi A, Gholami K. Reliability of Disk Diffusion Test Results for the Antimicrobial Susceptibility Testing of Nosocomial Gram-positive Microorganisms: Is E-test Method Better?. Iran J Pharm Res Spring, 2012; 11:559-563.

15. Zhang $P$, Zhang $X$, Brown J, Vistisen $D$, Sicree $R$, Shaw J. Global healthcare expenditure on diabetes for 2010 and 2030. Diabetes Res Clin Pract., 2010; 87:293-301.

16. Mendes JJ, Marques-Costa A, Vilela C, Neves J, Candeias N, Cavaco-Silva P. Clinical and bacteriological survey of diabetic foot infections in Lisbon. Diabetes Res Clin Pract. 2012; 95:153-61.

17. Werner G, Coque TM, Franz CM, Grohmann E, Hegstad K, Jensen L, van Schaik W, Weaver K. Antibiotic resistant enterococci-Tales of a drug resistance gene trafficker. Int J Med Microbiol., 2013; 303:360-798.

18. Higuita NIA, Huy MM. Enterococcal disease, epidemiology and implications for treatment. In: Gilmore MS, Clewell DB, Ike Y, Shankar N, editors. Enterococci: from commensals to leading causes of drug resistant infection. Boston: Mas- sachusetts Eye and Ear In rmary 2014; 1-15.

19. Dupre I, Zanetti S, Schito AM, Fadda G, Sechi LA. Incidence of virulence determinants in clinical Enterococcus faecium and Enterococcus faecalis isolates collected in Sardinia (Italy). J Med Microbiol., 2003; 52:491-498.

20. Gopinath R, Prakash M. Antibacterial activity of three medicinal plantsagainst clinically isolated multidrug resistant Enterococcus faecalis(MDRE). Int J Curr 
Microbiol App Sci., 2013; 2:6-14.

21. Wu M, Pan H, Leng W, Lei X, Chen L, and Liang Z. Distribution of Microbes and Drug Susceptibility in Patients with Diabetic Foot Infections in Southwest China. Journal of Diabetes Research, 2018; 1-9.

22. Rams TE, Feik D, Mortensen JE, Degener JE, van Winkelhoff AJ. Antibiotic susceptibility of periodontal Enterococcus faecalis. J. Periodontol, 2013; 84:102633.

23. Jia W, Gang Li, and Wang W. Prevalence and Antimicrobial Resistance of Enterococcus Species: A Hospital-Based Study in China. Int J Environ Res Public Health, 2014; 11:3424-3442.

24. Komiyama EY, Lepesqueur LS, Yassuda CG, Samaranayake LP, Parahitiyawa NB, Balducci I, Koga CY. Enterococcus Species in the Oral Cavity: Prevalence, Virulence Factors and Antimicrobial Susceptibility. Plos One, 2016; 0163001.

25. Anvarinejad M, Pouladfar G, Japoni A, Bolandparvaz S,
Satiary Z, Abbasi P, Mardaneh J. Isolation and Antibiotic Susceptibility of the Microorganisms Isolated from Diabetic Foot Infections in Nemazee Hospital, Southern Iran. Journal of Pathogens, 2015; ID:328796.

26. Citron DM, Goldstein EJ, Merriam CV, Lipsky BA, Abramson MA. Bacteriology of moderate-to-severe diabetic foot infections and in vitro activity of antimicrobial agents. J Clin Microbiol., 2007; 45:281928.

27. CLSI, Clinical and Laboratory Standards Institute. Performance standards for antimicrobial susceptibility testing; $20^{\text {th }}$ informational supplement. CLSI document M100-S21. Clinical and Laboratory Standards Institute, Wayne, Pa 2011.

28. Anvarinejad M, Japoni A, Rafaatpour N, Mardaneh J, Abbasi P, Amin Shahidi M. Burn Patients Infected With Metallo-Beta-LactamaseProducing Pseudomonas aeruginosa: Multidrug-Resistant Strains. Arch Trauma Res., 2014; 3:181-182. 